Pacific

Journal of

Mathematics

PERIODIC ORBITS OF HAMILTONIAN FLOWS NEAR SYMPLECTIC EXTREMA

VikTor L. Ginzburg And Ely Kerman 


\title{
PERIODIC ORBITS OF HAMILTONIAN FLOWS NEAR SYMPLECTIC EXTREMA
}

\author{
VikTor L. GinzBurg And Ely Kerman
}

\begin{abstract}
For Hamiltonian flows we establish the existence of periodic orbits on a sequence of level sets approaching a Bottnondegenerate symplectic extremum of the Hamiltonian. As a consequence, we show that a charge on a compact manifold with a nondegenerate (i.e., symplectic) magnetic field has periodic orbits on a sequence of energy levels converging to zero.
\end{abstract}

\section{Introduction.}

In the early seventies, Alan Weinstein proved the following result which was subsequently reproved by Jurgen Moser (using different methods) and is now known as the Weinstein-Moser Theorem (see [We1, We3, Mo]).

Theorem 1.1 (Weinstein-Moser). Let $H$ be a smooth function on a symplectic manifold of dimension 2n. Then the Hamiltonian flow of $H$ has at least $n$ periodic orbits on all level sets sufficiently close to a nondegenerate extremum point of $H$.

In this paper, we attempt to extend this result from extremal points to higher dimensional extrema of the Hamiltonian. In particular, we consider the case of symplectic extremal submanifolds. More precisely, let $H$ be a smooth function on a symplectic manifold $(W, \Omega)$ such that $H$ reaches an extremum at a compact symplectic Bott-nondegenerate submanifold $M^{2 l} \subset$ $W^{2 n}$. We prove the following:

Theorem 1.2. The Hamiltonian flow defined on $(W, \Omega)$ by the function $H$ has at least one periodic orbit on a sequence of energy levels converging to $M$.

In comparing these theorems, we see that here the existence of periodic orbits for all sufficiently close level sets is weakened to existence on a sequence of level sets approaching $M$. We also note that the lower bound for the number of periodic orbits is replaced by simple existence. It is unlikely that this result is sharp. (The uncertainty as to where the true boundaries of such existence results should lie is indicative of a lack of examples in this area.) However, other existence results of this kind have recently been obtained, see [Po] (Theorem 1.7 below) and [Ma]. Moreover, a result that is 
similar in strength to the Weinstein-Moser Theorem can be proved if one imposes certain compatibility conditions on $\Omega$ and the Hessian of $H$ on $M$. Specifically, in $[\mathbf{K e}]$ it is shown that under such assumptions there are at least $\mathrm{CL}(M, \mathbb{Q})+(n-l)$ periodic orbits on all level sets sufficiently close to $M$. Here $\operatorname{CL}(M, \mathbb{Q})$ denotes the cup-length of $M$ over $\mathbb{Q}$.

1.1. Symplectic magnetic flows. The question addressed here is motivated further by the following interesting set of examples. Let $(M, \omega)$ be a compact symplectic manifold and $g$ a Riemannian metric on $M$. Consider the Hamiltonian flow defined on $T^{*} M$ by the kinetic energy Hamiltonian

$$
\begin{aligned}
H_{g}: T^{*} M & \rightarrow \mathbb{R} \\
(q, p) & \mapsto\|p\|_{g^{-1}}^{2}
\end{aligned}
$$

and the twisted symplectic from $d \lambda+\pi^{*} \omega$. Here $\pi: T^{*} M \rightarrow M$ is the projection map and $\lambda$ is the canonical Liouville one-form. These flows describe the motion of a charged particle in a nondegenerate magnetic field and will be referred to as symplectic magnetic flows. The zero section of $T^{*} M$ is a symplectic minimum of $H_{g}$ and in the context of the result above we are concerned with the existence of periodic orbits on low energy levels. For such flows, Theorem 1.2 implies the following result.

Theorem 1.3. For any symplectic form $\omega$ and metric $g$ on $M$, the corresponding symplectic magnetic flow has periodic orbits on a sequence of low energy levels converging to zero.

To the knowledge of the authors, this is the most general existence result for symplectic magnetic flows. However, much stronger results have been established in a variety of different cases.

Theorem 1.4 ([Ar, Gi1] $]$. Let $M$ be a surface of genus $k$. Then for any choice of $\omega$ and $g$, the corresponding symplectic magnetic flow has at least three periodic orbits on all sufficiently low energy levels and at least $2 k+2$ if they are nondegenerate.

Theorem $1.5([\mathbf{K e}, \mathbf{G K}])$. Let the metric $g$ be of the form $\omega(\cdot, J \cdot)$ for some almost complex structure $J$ on $M$. Then the corresponding symplectic magnetic flow has at least $\mathrm{CL}(M, \mathbb{R})+l$ periodic orbits on all sufficiently low energy levels and at least $\mathrm{SB}(M)$ if all orbits are nondegenerate. Here $\mathrm{SB}(M)$ denotes the sum of Betti numbers of $M$.

In fact, the construction above produces a Hamiltonian flow for any manifold $M$, closed two-form $\omega$ and metric $g$, i.e., $\omega$ can be degenerate. We call these flows magnetic flows and refer the reader to $[\mathbf{G i 2}]$ for a more detailed discussion of them and further references. The following are recent results for magnetic flows which also hold in the symplectic case. 
Theorem $1.6([\mathbf{G K}])$. For any closed two-form $\omega$ and metric $g$ on $\mathbb{T}^{n}$, the corresponding magnetic flow has periodic orbits on almost all energy levels. (In fact, bounded neighborhoods of the zero section have finite Hofer-Zehnder capacity.)

Theorem 1.7 ([Ma, Po]). For any metric on $M$ and any nonzero weaklyexact $^{1}$ two-form, the corresponding magnetic flow has contractible periodic orbits on a sequence of energy levels converging to zero.

These last two theorems represent interesting applications of tools from other areas of symplectic topology to the existence question. The first result is a direct application of the work on the Hofer-Zehnder capacity in [FHV] and $[\mathbf{J i}]$. The second result is proved for $M=\mathbb{T}^{n}$ by Polterovich using Hofer's metric on the space of Hamiltonian diffeomorphisms (see $[\mathbf{P o}]$ ). In particular, he utilizes the relation between the nonminimizing geodesics of Hofer's metric and the existence of contractible periodic orbits (see $[\mathbf{L M}]$ ). This work is then extended to the more general form, as stated here, by Macarini in $[\mathbf{M a}]$.

\section{Limiting dynamics and the variational problem.}

2.1. The variational problem. Before starting the proof of Theorem 1.2, we recall the variational framework for proving the existence of periodic orbits of the Hamiltonian flow of a function $H$ on a symplectic manifold $(W, \Omega)$. First we choose a suitable class of loops in $W$, say the Fréchet manifold of smooth loops, $C^{\infty}\left(S^{1}, W\right)$. Then on $C^{\infty}\left(S^{1}, W\right)$ we consider the one-form $\mathfrak{F}$ which takes $v$, an element of the tangent space at $\sigma \in$ $C^{\infty}\left(S^{1}, W\right)$, to

$$
\mathfrak{F}(v)=\int_{0}^{1} \Omega(\sigma(s))(\dot{\sigma}(s), v(s)) d s-\int_{0}^{1} d H(\sigma(s))(v(s)) d s .
$$

It is clear from the least action principle that the zeroes of $\mathfrak{F}$ on $C^{\infty}\left(S^{1}, W\right)$ are periodic orbits of the Hamiltonian flow with period equal to one.

At this point we are faced with several difficulties. The first among them is the fact that the variational problem is far more tractable if $\mathfrak{F}$ is exact. For then we may look for the critical points of a functional instead of the zeros of a one-form. As well, there need not exist closed orbits with period equal to one. Finally, as stated, the variational approach does not allow us to search for periodic orbits on a fixed level set of $H$.

The analysis of the limiting dynamics given below suggests that the search for periodic orbits on level sets near $M$ can be restricted to the subset of small loops in $C^{\infty}\left(S^{1}, W\right)$ that lie close to $M$. In making this restriction, we are able to overcome the first problem by finding an action functional

\footnotetext{
${ }^{1}$ Recall that a form $\omega$ is called weakly-exact if $\omega$ is closed and $\left.[\omega]\right|_{\pi_{2}(M)}=0$.
} 
whose derivative is equal to $\mathfrak{F}$ on this subset. The restriction also allows us to considerably simplify the analytic setting by using the method developed by Weinstein in [We4] to prove the Arnold conjecture for $C^{0}$-small Hamiltonians. The other difficulties are surmounted by adapting a set of techniques developed by Viterbo, and Hofer and Zehnder in [HZ1, HZ2, HZ3, Vi]. Namely, we modify the function $H$ (and hence our functional) to force any periodic orbits on the desired energy levels to have positive action. Then we show that the new action functional has critical points with positive action by using a "linking argument" as in [HZ1, HZ2, HZ3].

2.2. The limiting dynamics. First, we consider the case when $M$ is an extremal point of the function $H: W^{2 n} \rightarrow \mathbb{R}$ and we recall briefly how one begins to look for periodic orbits on the level sets near this point. Darboux's theorem allows us to work in a neighborhood of the origin in $\mathbb{R}^{2 n}$ with the canonical symplectic form $\Omega_{0}$. Here the function looks like

$$
H(z)=\frac{1}{2} H_{z z}(0) z^{2}+O\left(z^{3}\right)
$$

and we may assume that the origin is a local minimum so that the quadratic form $H_{z z}(0)$ is positive definite. The Hamiltonian vector field corresponding to $H$ is denoted by $X_{H}$ and is defined by

$$
i_{X_{H}} \Omega_{0}=d H .
$$

Let

$$
H_{\epsilon}(z)=\epsilon^{-2} H(\epsilon z)=\frac{1}{2} H_{z z}(0) z^{2}+O(\epsilon) .
$$

For $\epsilon \neq 0$, the rescaling map $z \mapsto \epsilon z$ takes the flow of $X_{H}$ on $\left\{H=\epsilon^{2}\right\}$ to the flow of the Hamiltonian vector field $X_{H_{\epsilon}}$ on $\left\{H_{\epsilon}=1\right\}$. Thus, we may instead look for periodic orbits for this flow.

The vector field $X_{H_{\epsilon}}$ is defined by

$$
i_{X_{H_{\epsilon}}} \Omega_{0}=d H_{\epsilon}
$$

and we see that it is a Hamiltonian perturbation of the linear Hamiltonian vector field $X_{0}$ given by the equation

$$
i_{X_{0}} \Omega_{0}=H_{z z}(0) z \text {. }
$$

Since $H_{z z}(0)$ is positive definite, there exists a change of variable $z \mapsto y$ which preserves $\Omega_{0}$ and puts $H_{z z}(0)$ in the form

$$
H_{z z}(0) y^{2}=\sum_{i=1}^{n} \frac{a_{i}}{2}\left(y_{i}^{2}+y_{i+n}^{2}\right),
$$

see $[\mathbf{H Z 3}, \S 1.7]$. In these coordinates it becomes clear that $X_{0}$ describes the quasiperiodic motion of $n$ uncoupled harmonic oscillators with frequencies $a_{i}$. The problem then reduces to showing that the periodic "normal modes" 
of $X_{0}$ persist under the perturbation $X_{H_{\epsilon}}$ (see $[\mathbf{L y}, \mathbf{M o}$, We1, We3] and $[\mathbf{F R}])$.

Next we consider the case when $M$ is a symplectic extremal submanifold. We will rescale globally in the normal directions to $M$ and show that we still get a well-defined and useful limiting vector field.

Set $\left.H\right|_{M}=0$. The Tubular Neighborhood Theorem allows us to assume that, for sufficiently small $\epsilon>0$, the level sets $\left\{H=\epsilon^{2}\right\}$ lie in a neighborhood of the zero section in the total space of a normal bundle $N$ to $M$. Accordingly, we may replace the manifold $W$ by this neighborhood which we will still denote by $W$. We choose the normal bundle to be $(T M)^{\Omega}$, the symplectic orthogonal complement to TM. By Weinstein's Symplectic Neighborhood Theorem (see [We2]) we may also assume that $\Omega$ restricts to the fibres in $(T M)^{\Omega} \cap W$ as a constant linear symplectic form, $\Omega^{N}$. Thus, the level sets of interest lie in a symplectic vector bundle which is also equipped with a fibrewise positive-definite quadratic form $d_{N}^{2} H$, given by the Hessian of $H$ in the normal directions to $M$. As above, there exist coordinates $\left\{y_{i}(x)\right\}_{i=1}^{2(n-l)}$ in each fibre $E_{x}$ such that $\Omega^{N}(x)$ is the canonical symplectic form on $\mathbb{R}^{2(n-l)}$ and

$$
d_{N}^{2} H(x) y^{2}=\sum_{i=1}^{(n-l)} \frac{a_{i}(x)}{2}\left(y_{i}^{2}+y_{i+(n-l)}^{2}\right) .
$$

Note that in general these coordinates are not unique and cannot be chosen to depend smoothly or even continuously on $x$. However, the eigenvalues $a_{i}(x)$ of $d_{N}^{2} H(x)$ with respect to $\Omega^{N}(x)$ are well-defined.

Starting with the Hamiltonian dynamical system defined on $W \subset N$ by

$$
i_{X_{H}} \Omega=d H
$$

we let $\Phi: N \rightarrow N$ be the global fibrewise dilation by a factor of $\epsilon$, and set

$$
X_{\epsilon}:=\Phi_{*}^{-1} X_{H}
$$

and

$$
\widetilde{\Omega}_{\epsilon}:=\epsilon^{-2} \Phi^{*} \Omega .
$$

After this rescaling the new Hamiltonian dynamical system is given by

$$
i_{X_{\epsilon}} \widetilde{\Omega}_{\epsilon}=d\left(\epsilon^{-2} \Phi^{*} H\right) .
$$

Lemma 2.1. As $\epsilon \rightarrow 0, X_{\epsilon}$ approaches a fibrewise, quasiperiodic vector field $X_{0}$. In particular, in each fibre $E_{x}$, the vector field $X_{0}$ is the (linear) Hamiltonian vector field of the positive-definite form $d_{N}^{2} H(x)$ with respect to the symplectic form $\Omega^{N}(x)$.

Proof. One can check that the fibre components of the $\widetilde{\Omega}_{\epsilon}$ are independent of $\epsilon$ and equal to $\Omega^{N}$. However, the limit of the $\widetilde{\Omega}_{\epsilon}$ as $\epsilon \rightarrow 0$ is not defined, 
i.e., in coordinates, all the terms with components along the base blow up in the limit. Using the bundle isomorphisms

$$
\left(\widetilde{\Omega}_{\epsilon}\right)^{b}: T W \rightarrow T^{*} W
$$

defined by each of the nondegenerate forms $\widetilde{\Omega}_{\epsilon}$, we can construct the dual bivectors $-\widetilde{\Omega}_{\epsilon}^{-1} \in \Lambda^{2}(T W)$. (We include the negative sign because if we associate to $\widetilde{\Omega}_{\epsilon}(m)$ a nondegenerate skew-symmetric matrix, then to the dual bivector at $m$ we associate the negative inverse of this matrix.) These bivectors are nondegenerate Poisson structures and we may rewrite Equation (3) as

$$
X_{\epsilon}=-\widetilde{\Omega}_{\epsilon}^{-1}\left[d\left(\epsilon^{-2} \Phi^{*} H\right)\right] .
$$

In contrast to the $\widetilde{\Omega}_{\epsilon}$ the Poisson structures $\widetilde{\Omega}_{\epsilon}^{-1}$ do have a well-defined limit, $\left(\Omega^{N}\right)^{-1}$. This is a degenerate Poisson structure whose symplectic leaves are the fibres of $N$. As well, for $\epsilon \rightarrow 0$ we have $\epsilon^{-2} \Phi^{*} H \rightarrow d_{N}^{2} H$. Hence,

$$
\begin{aligned}
X_{0} & :=\lim _{\epsilon \rightarrow 0} X_{\epsilon} \\
& =-\left(\Omega^{N}\right)^{-1}\left[\left(d^{N}\left(d_{N}^{2} H\right)\right],\right.
\end{aligned}
$$

where $d^{N}$ denotes the exterior derivative with respect to just the fibre variables. This can be rewritten as

$$
i_{X_{0}} \Omega^{N}=d^{N}\left(d_{N}^{2} H\right) .
$$

Indeed, this equation defines the limiting vector field $X_{0}$ globally and we note that the convergence of $X_{\epsilon}$ to $X_{0}$ is $C^{k}$ for any $k$.

By Equation (2) the flow of $X_{0}$ is fibrewise quasiperiodic and we have at least $(n-l)$ periodic orbits in each fibre.

By Lemma 2.1, the flow on the level $\left\{H=\epsilon^{2}\right\}$ can be viewed (up to parameterization) as a small (Hamiltonian) perturbation of the quasiperiodic flow of $X_{0}$ on $\left\{d_{N}^{2} H=1\right\}$. Hence, one may expect that under this perturbation the set of periodic orbits of $X_{0}$ on $\left\{d_{N}^{2} H=1\right\}$ (called the normal modes of $X_{0}$ following [We3]) splits into periodic orbits on $\left\{H=\epsilon^{2}\right\}$ whose number is bounded from below by the cup-length or the sum of Betti numbers of this set. These invariants depend on the pair of fibrewise forms $d_{N}^{2} H$ and $\Omega^{N}$ but should be greater than or equal to the corresponding invariants of $M$.

When the eigenvalues $a_{i}(x)$ do not bifurcate as functions of the parameter $x \in M$, the set of normal modes of $X_{0}$ is an orbifold, $[\mathbf{K e}]$. In this case the perturbative analysis can indeed be carried out by adapting Moser's method, see [Bo, Mo]. This leads to a lower bound on the number of periodic orbits in terms of the cup-length, $[\mathbf{K e}]$. Furthermore, when for every $x$ the eigenvalues $a_{i}(x)$ are equal to each other, a lower bound in terms of the sum of Betti numbers of $M$ has also been obtained in $[\mathbf{G K}]$. (This is the case, 
for example, when $\operatorname{codim} M=2$. In particular, it is true for symplectic magnetic flows on surfaces, [Gi1, Gi3]. The condition is also satisfied for symplectic magnetic flows in higher dimensions when $g=\omega(\cdot, J \cdot)$ for some almost complex structure $J$ on $M$, [Ke].)

In general, this perturbative approach encounters serious difficulties arising from the fact that the set of normal modes of $X_{0}$ may fail to be a manifold or an orbifold. In this work the limiting dynamics is used only as motivation.

\section{Simplification of the variational problem.}

Expecting some of the normal modes of the limiting vector field $X_{0}$ to always persist under the Hamiltonian perturbation $X_{\epsilon}$, we will restrict our search for periodic orbits to small loops near $M$. This will considerably simplify the original variational problem.

To begin with, we fix some geometric structure on $\left.T W\right|_{M}$. Let $J$ be an almost complex structure that is compatible with $\Omega$. This yields the Riemannian metric $g_{J}=\Omega(\cdot, J \cdot)$ on $W$. With the splitting

$$
\left.T W\right|_{M}=T M \oplus(T M)^{\Omega},
$$

we then have the decomposition

$$
\left(T_{m} W, \Omega, J, g_{J}\right)=\left(T_{m} M \oplus\left(T_{m} M\right)^{\Omega}, \omega_{T} \oplus \omega_{N}, J_{T} \oplus J_{N}, g_{T} \oplus g_{N}\right)
$$

where the subscripts $T$ and $N$ denote tangential and normal components, respectively. With respect to this splitting we will write $z=(x, y)$ for $z \in T_{m} W$, where $x \in T_{m} M$ and $y \in\left(T_{m} M\right)^{\Omega}$.

3.1. A Darboux family. Following [We4], we note the existence of a Darboux family for $M \subset W$. This a parameterized version of a Darboux chart. It consists of a neighborhood $U$ of the zero section in $\left.T W\right|_{M}$ and a mapping

$$
\Phi: U \longrightarrow W
$$

onto a neighborhood $V$ of $M \subset W$ such that the following conditions hold.

1. $U_{m}=U \cap T_{m} W$ contains the origin.

2. $\Phi_{m}=\left.\Phi\right|_{U_{m}}$ is a symplectomorphism from $\left(U_{m}, \Omega(m)\right)$ to $\left(V_{m}, \Omega\right)$, where $V_{m}$ is an open neighborhood of $m \in W$.

3. $\Phi_{m}(0)=m$ and $D_{0} \Phi_{m}$ is the identity.

In addition, we may assume that all neighborhoods $U_{m}$ are open balls of a fixed radius with respect to $g_{J}$. For sufficiently small $\epsilon>0$, we may also assume that the level set $\left\{H=\epsilon^{2}\right\}$ lies in $V$.

3.2. A new loop space. Denote by $C_{0}^{\infty}\left(S^{1}, V\right)$ the open subset of $C^{\infty}\left(S^{1}\right.$, $W$ ) consisting of small loops contained in $V$. This is an open neighborhood of the constant loops in $V$. Based on our analysis of the limiting dynamics, this is also where we expect to find low energy periodic orbits. 
Now, let $\Lambda_{m}$ be the space of $C^{\infty}$ loops in $T_{m} W$ whose projections to $T_{m} M$ have zero mean, and consider the Fréchet space bundle

$$
\Lambda=\bigcup_{m \in M} \Lambda_{m}
$$

The map $\Phi$ pulls back $C_{0}^{\infty}\left(S^{1}, V\right)$ onto an open neighborhood $\mathcal{U}$ of the zero section in $\Lambda$, which in each fibre $\Lambda_{m}$ consists of loops contained in $U_{m}$. This follows from the inverse function theorem and, put another way, is essentially the fact that any small loop in $M$ has a unique mean value in $M$ with respect to the map $\Phi$. To be more precise, for every $\sigma \in C_{0}^{\infty}\left(S^{1}, V\right)$, there exists a unique $m \in M$ such that $\sigma=\Phi_{m}(z)$ for some loop $z \in \Lambda_{m}$ which takes values in $U_{m}$.

Since $\Omega$ is exact in a neighborhood of any of the loops in $C_{0}^{\infty}\left(S^{1}, V\right)$, the differential form $\left.\mathfrak{F}\right|_{C_{0}^{\infty}\left(S^{1}, V\right)}$ is exact and so is its pullback by $\Phi$. (Indeed, the second term in (1) is always exact and the symplectic area of a small disc bounded by the loop can be taken as a primitive of the first term.) We denote the primitive of $\Phi^{*}\left(\left.\mathfrak{F}\right|_{C_{0}^{\infty}\left(S^{1}, V\right)}\right)$ by $F^{0}$ and remark that $F^{0}(m, z)=F_{m}^{0}(z)$, where $F_{m}^{0}$ is the restriction to $\mathcal{U} \cap \Lambda_{m}$ and is given by

$$
F_{m}^{0}(z)=\int_{0}^{1} \frac{1}{2} g_{J}(m)(-J(m) \dot{z}, z) d t-\int_{0}^{1} H\left(\Phi_{m}(z(t))\right) d t .
$$

This is just the standard action functional for the Hamiltonian $\Phi_{m}^{*} H$ which is defined on the open subset $U_{m}$ of the symplectic vector space $\left(T_{m} W, \Omega(m)\right)$. In particular, as above, the first term is the symplectic area of the disc bounded by $z$ in this space.

We have thus simplified the original variational problem to that of finding a critical point of the functional $F^{0}$ in $\mathcal{U}$ (a neighborhood of the zero section in the Fréchet space bundle $\Lambda$ ).

\section{An outline of the proof.}

4.1. Step 1. First we utilize the freedom to choose another Hamiltonian $\widetilde{H}: W \rightarrow \mathbb{R}$ that shares the level set $\left\{H=\epsilon^{2}\right\}$ with $H$. In fact, we make our changes to the pullbacks $\Phi_{m}^{*} H$ in such a way that each new function $h_{m}$ keeps the level set $\left\{\Phi_{m}^{*} H=\epsilon^{2}\right\} \subset T_{m} W$ and is equal to the pull back, by $\Phi_{m}$, of a new global Hamiltonian $\widetilde{H}$ defined on $V \subset W$. We then consider the functional $F(m, z)=F_{m}(z)$ defined on $\mathcal{U} \subset \Lambda$ by

$$
F_{m}(z)=\int_{0}^{1} \frac{1}{2} g_{J}(m)(-J(m) \dot{z}, z) d t-\int_{0}^{1} h_{m}(z(t)) d t .
$$

Choosing extensions of the maps $h_{m}$ so that the functional $F$ is defined on all of $\Lambda$ we then prove 
Lemma 4.1. The choices and extensions above can be made in such a way that any critical point $z_{c}$ of $F$ on $\Lambda$, satisfying $F\left(z_{c}\right)>0$, corresponds to a periodic orbit of the original system on $\left\{H=\epsilon^{2}+\rho \frac{\epsilon^{2}}{4}\right\}$ for some $\rho \in[-\epsilon, \epsilon]$.

4.2. Step 2. We extend the domain of definition of $F$ to be the Hilbert space bundle

$$
\widetilde{\Lambda}=\bigcup_{m \in M} \widetilde{\Lambda}_{m}
$$

where $\widetilde{\Lambda}_{m}$ is the space of $H^{\frac{1}{2}}$ loops in $T_{m} W$ whose projections to $T_{m} M$ have zero mean.

Theorem 1.2 will then follow from:

Lemma 4.2. There exists a critical point $z_{c} \in \Lambda \subset \widetilde{\Lambda}$ of $F$ such that $F\left(z_{c}\right)>0$.

\section{Step 1.}

5.1. A new Hamiltonian and functional. In looking for periodic orbits on the level set $\left\{H=\epsilon^{2}\right\}$ we may replace $H$ by any other function which shares this level set. Here we construct such a function which also shares with $H$ all the level sets close to $\left\{H=\epsilon^{2}\right\}$. This is accomplished by altering (and extending) the pullbacks $\Phi_{m}^{*} H$ into a family of functions $h_{m}: T_{m} W \rightarrow \mathbb{R}^{+}$which is smooth in $m$ and satisfies the following conditions:

1. There is a function $\widetilde{H}: V \subset W \rightarrow \mathbb{R}$ such that $\Phi_{m}^{*} \widetilde{H}=\left.h_{m}\right|_{U_{m}}$ for all $m \in M$ and $\widetilde{H}$ shares the level sets $\left\{H=\epsilon^{2}+\rho \frac{\epsilon^{2}}{4}\right\}$ with $H$, for all $\rho \in[-\epsilon, \epsilon]$.

2. All the $h_{m}=0$ on an open neighborhood of $T_{m} M \times\{0\} \subset T_{m} W$.

3. Let $Q_{m}(z)=\frac{q}{2}\|y\|_{m}^{2}$ for some positive $q$ to be specified later. Then $\left\|\nabla h_{m}(z)-\nabla Q_{m}(z)\right\|_{m}$ is bounded and $h_{m}=Q_{m}$ for large $\|y\|_{m}$.

4. For the functional $F$ defined in (5), a critical point $z_{c}$ with $F\left(z_{c}\right)>0$ corresponds to a periodic orbit of $X_{H}$ on $\left\{H=\epsilon^{2}+\rho \frac{\epsilon^{2}}{4}\right\}$ for some $\rho \in[-\epsilon, \epsilon]$.

Remark 5.1. The constant $q>0$ is chosen so that $q$ is not an even integer and $q$ is greater than a certain constant depending only on $W$ and $M$. The assumption that $q$ is not an even integer is crucial in verifying the PalaisSmale condition for the functional $F$ (Claim 6.4). The lower bound for $q$ is essential in Proposition 6.6; see also Remark 6.7.

5.1.1. Construction of the $h_{m}$. For $\rho \in[-1,1]$ and for each $m \in M$ let

$$
S_{\rho, m}=\left\{\phi_{m}^{*} H=\epsilon^{2}+\rho \frac{\epsilon^{2}}{4}\right\} \subset U_{m} .
$$


Extend the hypersurfaces $S_{\rho, m}$ outside $U_{m}$ by smoothly and quickly joining them to the hypersurfaces

$$
\left\{(x, y) \in T_{m} W \mid\|y\|_{m}=\epsilon^{2}+\rho \frac{\epsilon^{2}}{4}\right\} .
$$

We still refer to these extended hypersurfaces as the $S_{\rho, m}$ and note that they will be level surfaces of our new functions $h_{m}$. Denote the union of these hypersurfaces in $T_{m} W$ by

$$
C_{m}=\bigcup_{\rho \in[-1,1]} S_{\rho, m}
$$

Set

$$
\gamma_{m}=\max _{(x, y) \in S_{1, m}}\|y\|_{m} \quad \text { and } \quad \gamma=\max _{m \in M} \gamma_{m} .
$$

Fixing $q$ as in Remark 5.1, we choose constants $r$ and $b$ such that

$$
\gamma<r<2 \gamma \quad \text { and } \quad \frac{q}{2} \pi r^{2}<b<q \pi r^{2} .
$$

We then use the following smooth functions to specify the behavior of $h_{m}$ in $C_{m}$ and asymptotically in the normal directions. Let $f \in C^{\infty}\left([-1,1], \mathbb{R}^{+}\right)$ have the properties

$$
f(s)= \begin{cases}0 & \text { for } s \in(-1,-\epsilon] \\ b & \text { for } s \in[\epsilon, 1)=b\end{cases}
$$

and

$$
f^{\prime}(s)>0 \text { for } \quad-\epsilon<s<\epsilon .
$$

Also, let $g \in C^{\infty}\left((0, \infty), \mathbb{R}^{+}\right)$satisfy

$$
g(s)= \begin{cases}b & \text { for } s \leq r \\ \frac{q}{2} \pi s^{2} & \text { for large } s\end{cases}
$$

and

$$
g(s) \geq \frac{q}{2} \pi s^{2} \quad \text { and } \quad 0<\dot{g}(s) \leq q \pi s \quad \text { for } \quad s>r .
$$

Note that $T_{m} W \backslash C_{m}$ has two connected components, $A_{m}$ and $B_{m}$, where $B_{m}$ is the open set containing $T_{m} M \times\{0\} \subset T_{m} W$. Finally, we set

$$
h_{m}(z)= \begin{cases}0 & \text { if } z \in B_{m} \\ f(\rho) & \text { if } z \in S_{\rho, m} \text { for }-1<\rho<1 \\ b & \text { if } z \in A_{m} \text { and }\|y\|_{m} \leq r \\ g\left(\|y\|_{m}\right) & \text { if }\|y\|_{m} \geq r .\end{cases}
$$

(See Figure 5.1 below.) The functions $h_{m}$ defined in this way clearly satisfy Conditions 1, 2 and 3 as stated above. 


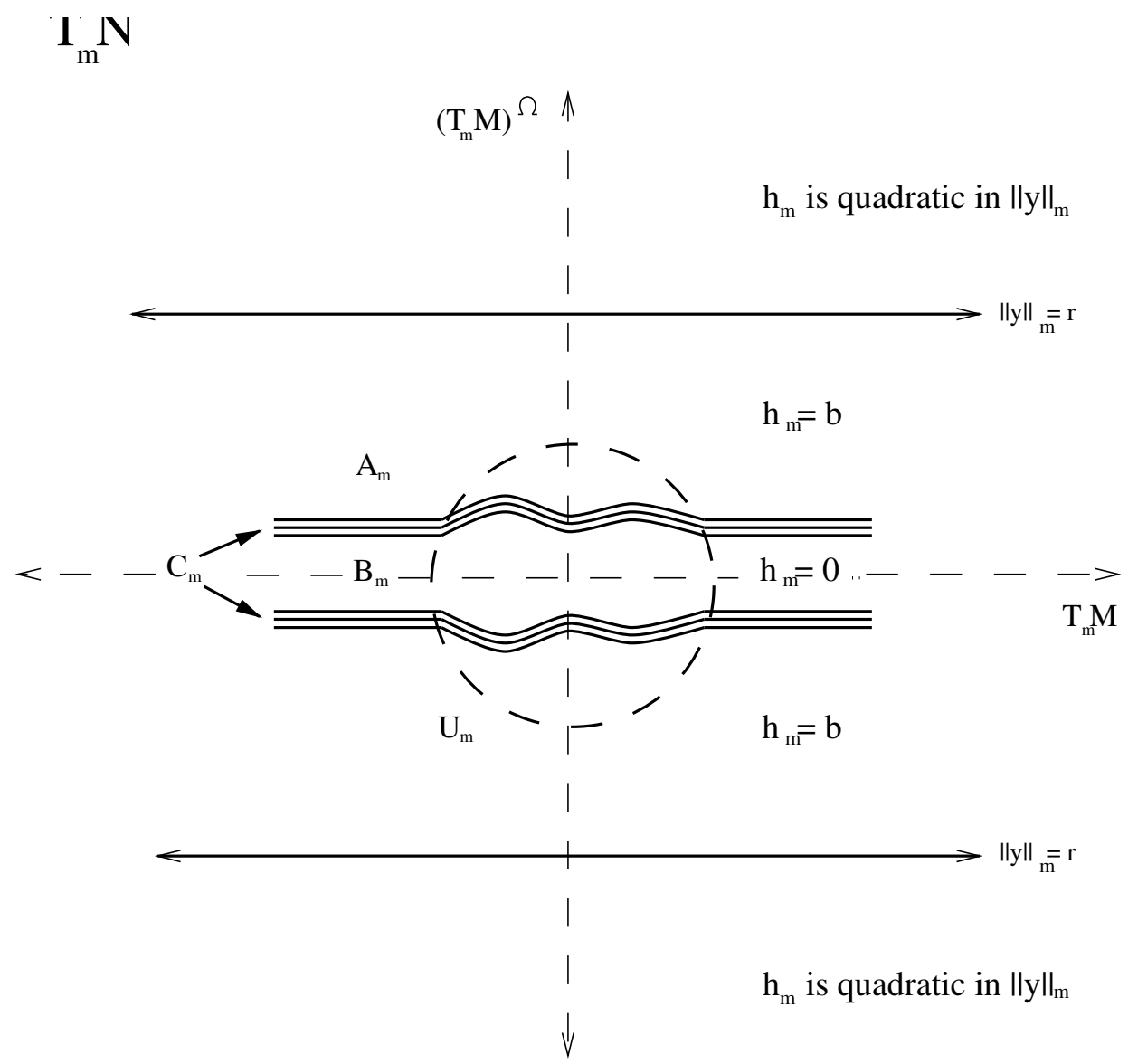

Figure 5.1. The functions $h_{m}$.

The choices of $f$ and $g$ also yield the following inequalities which will be used later:

$$
\begin{gathered}
-b+\frac{q}{2} \pi\|y\|_{m}^{2} \leq h_{m}(z) \leq \frac{q}{2} \pi\|y\|_{m}^{2}+b, \\
\left\|\nabla h_{m}(z)\right\|_{m} \leq c_{1}\|z\|_{m} \quad \text { for some } \quad c_{1} \in \mathbb{R}^{+} .
\end{gathered}
$$

The second of these is the most crucial and follows from the inequality

$$
\left\|\nabla_{N} h_{m}(z)\right\|_{m} \leq q \pi\|y\|_{m} \quad \text { for } \quad\|y\|_{m} \geq r
$$

coupled with the facts that $h_{m}(0)=0$ and that $\nabla_{T} h_{m}$ has compact support.

Remark 5.2. Each function $h_{m}$ is of the type considered in [Gi3]. They differ from those in $[\mathbf{V i}]$ and $[\mathbf{H Z 1}]$ in that the level sets $S_{\rho, m}$ are not compact and the functions are asymptotically quadratic only in the normal directions. 
5.2. Locating critical points with positive action. We now prove that the functions $h_{m}$ have property 4 .

Claim 5.3. A critical point of $F_{m}$, say $z(t)=x(t)+y(t)$, satisfying $F_{m}(z)>$ 0 must lie in $S_{\rho, m} \cap U_{m}$ for some $\rho \in[-\epsilon, \epsilon]$.

Proof. First we show that $F_{m}(z)>0$ implies that $z \in S_{\rho, m}$ for some $\rho \in$ $[-\epsilon, \epsilon]$. If $z(t)$ is a constant solution then

$$
F_{m}(z)=-\int_{0}^{1} h_{m}(z(t)) d t \leq 0
$$

since $h_{m} \geq 0$. Hence, we only have to discount those critical points with $\|y(t)\|_{m} \geq r$ for some $t \in[0,1]$. In fact, because $h_{m}$ depends only on the fibre variable past $r$, we get $\|y(t)\|_{m}=\|y(0)\|_{m}$ for all $t$ in $[0,1]$. When $\|y(t)\|_{m} \geq r$, we also have

$$
\nabla h_{m}(z(t))=\dot{g}\left(\|y(0)\|_{m}\right) \frac{y(t)}{\|y(0)\|_{m}} .
$$

Hence,

$$
\begin{aligned}
F_{m}(z) & =\int_{0}^{1}\left(\frac{1}{2} g_{J}(m)(-J(m) \dot{z}(t), z(t))-h_{m}(z(t))\right) d t \\
& =\int_{0}^{1}\left(\frac{1}{2} \dot{g}\left(\|y(0)\|_{m}\right)\|y(0)\|_{m}-h_{m}(y(0))\right) d t \\
& =\frac{1}{2} \dot{g}\left(\|y(0)\|_{m}\right)\|y(0)\|_{m}-h_{m}(y(0)) \\
& \leq \frac{1}{2} \dot{g}\left(\|y(0)\|_{m}\right)\|y(0)\|_{m}-\frac{q}{2} \pi\|y(0)\|_{m}^{2} \\
& \leq 0
\end{aligned}
$$

The last two inequalities follow from the fact that

$$
h_{m}(x, y)=g\left(\|y\|_{m}\right) \geq \frac{q}{2} \pi\|y\|_{m}^{2}
$$

for $\|y\|_{m} \geq r$, and $\dot{g}(s) \leq q \pi s$.

Next we show that $x(t) \subset U_{m} \cap T_{m} M$. A critical point of $F_{m}$ is a oneperiodic solution of the Hamiltonian dynamical system defined on $T_{m} W$ by $h_{m}$ and $\Omega(m)$. Thus, $x(t)$ has period one and because of our splitting of $\left.T W\right|_{M}$ it satisfies

$$
\dot{x}(t)=J_{T}(m) \nabla_{T} h_{m}(z(t)) .
$$

Now, since $x(t)$ has zero mean,

$$
x(t)=\int_{0}^{1}(x(s)-x(t)) d s .
$$


Hence,

$$
\begin{aligned}
\|x(t)\|_{m} & \leq \int_{0}^{1}\|x(s)-x(t)\|_{m} d s \\
& \leq \sup _{s \in[0,1]}\|\dot{x}(s)\|_{m} \\
& \leq\left\|J_{T}(m)\right\|_{m} \sup _{z \in T_{m} W}\left\|\nabla_{T} h_{m}(z)\right\|_{m} .
\end{aligned}
$$

It is easily checked for our choice of $f$ that $\left\|\nabla_{T} h_{m}(z)\right\|_{m}$ is of order $\epsilon$ for all $z \in C_{m}$. Hence, $x(t)$ remains sufficiently close to the origin and the proof of the claim is complete.

Since a critical point of $F$ on $\Lambda$ must also be a critical point of $F_{m}$ on $\Lambda_{m}$ for some $m \in M$, the claim yields Lemma 4.1.

Remark 5.4. At this point we can see why a stronger existence result is not attainable using these techniques. For example, consider what happens when we try to prove that there are periodic orbits on level sets arbitrarily close to a fixed level set $\left\{H=\epsilon^{2}\right\}$. As in the previous claim, we would like to distinguish any periodic orbits on the candidate level sets by forcing them to have positive action. Accordingly, we define the functions $h_{m}$ using a new function $f$ which switches from 0 to $b$ on an arbitrarily small neighborhood of zero, say $(-\delta, \delta)$. Unfortunately, when $\delta$ is too small, i.e., when we look for periodic orbits on level sets too close to $\left\{H=\epsilon^{2}\right\}$, we lose control of the size of $\left\|\nabla_{T} h_{m}(z)\right\|_{m}$. Consequently, given a critical loop $z \in \Lambda_{m}$ with positive action, we no longer know that $z$ lies within $U_{m}$. Some part of it may lie in the extended portions of $S_{\rho, m}$ and so $z$ no longer corresponds to a periodic orbit of our original system.

\section{Step 2.}

6.1. Extending the domain of $\mathbf{F}$. We extend the domain of the functional $F$ from the Fréchet space bundle $\Lambda$ to the Hilbert space bundle $\widetilde{\Lambda}$ defined below. This extension is motivated by the fact that $F$ has a simple form on $\widetilde{\Lambda}$ which allows us to easily verify that the negative gradient flow of $F$ has the properties necessary to employ Minimax techniques to detect critical points. In particular, we are able to extend the "linking argument" of Hofer and Zehnder for $\mathbb{R}^{2 n}$ (see $[\mathbf{H Z 1}, \mathbf{H Z 2}, \mathbf{H Z 3}]$ ) to our bundle $\left.T W\right|_{M}$ over $M$.

Remark 6.1. Alternatively, one can show that $F$ has a positive critical value by using the cohomological argument as in $[\mathbf{V i}]$ or [Gi3], combined with the reduction to finite dimensions from $[\mathbf{C Z}]$.

Let $\widetilde{\Lambda}$ be the Hilbert bundle over $M$ with fibres $\widetilde{\Lambda}_{m}$ consisting of $H^{\frac{1}{2}}$ loops in $T_{m} W$ whose projections to $T_{m} M$ have zero mean. We may consider $\widetilde{\Lambda}_{m}$ 
as the space of Fourier series

$$
z(t)=\sum_{k \in \mathbb{Z}} e^{k 2 \pi J(m) t} z_{k}
$$

with $z_{k}=x_{k}+y_{k} \in T_{m} W$ and $x_{0}=0$, which converge with respect to the norm \|\|$_{\frac{1}{2}, m}$ given by the inner product

$$
\left\langle z, z^{\prime}\right\rangle_{m}=g_{J}(m)\left(z_{0}, z_{0}^{\prime}\right)+2 \pi \sum_{k \in \mathbb{Z}}|k| g_{J}(m)\left(z_{k}, z_{k}^{\prime}\right) .
$$

This space clearly includes $\Lambda$ and is contained in the bundle of fibrewise $L^{2}$-loops.

The bundle $\widetilde{\Lambda}$ splits naturally in two ways. First there is the orthogonal splitting which in each fibre has the form

$$
\widetilde{\Lambda}_{m}=E_{m}^{-} \oplus E_{m}^{0} \oplus E_{m}^{+} .
$$

Here, the space $E_{m}^{-}$consists of the series with nonzero Fourier coefficients for $k<0$ only. The spaces $E_{m}^{0}$ and $E_{m}^{+}$are defined analogously. We also have the orthogonal splitting of the $\widetilde{\Lambda}_{m}$ into loops contained in $T_{m} M$ and loops contained in $\left(T_{m} M\right)^{\Omega}$. For example, $z(t)=x(t)+y(t)$ where the Fourier coefficients of $x(t)$ and $y(t)$ are contained in $T_{m} M$ and $\left(T_{m} M\right)^{\Omega}$, respectively. We denote this splitting by

$$
\widetilde{\Lambda}=E_{T} \oplus E_{N} .
$$

In considering the functional $F$ on the bundle $\widetilde{\Lambda}$, we focus first on the fibres where we have

$$
F_{m}(z)=\int_{0}^{1} \frac{1}{2} \widetilde{g}_{J}(m)(-J(m) \dot{z}, z) d t-\int_{0}^{1} h_{m}(z(t)) d t .
$$

With respect to the orthogonal splitting $z=z^{-}+z^{0}+z^{+}$it is straightforward to check that

$$
F_{m}(z)=\frac{1}{2}\left(\left\|z^{+}\right\|_{\frac{1}{2}, m}^{2}-\left\|z^{-}\right\|_{\frac{1}{2}, m}^{2}\right)-\int_{0}^{1} h_{m}(z(t)) d t
$$

and the $H^{\frac{1}{2}}$-gradient with respect to the fibre variables of $\widetilde{\Lambda}_{m}$ is

$$
\nabla F_{m}(z)(v)=g_{J}(m)\left(z^{+}-z^{-}, v\right)-\int_{0}^{1} g_{J}(m)\left(\nabla h_{m}(z), v\right) d t .
$$

The total gradient flow of $F$ is actually comprised of the gradient flows of the $F_{m}$ on the fibres $\widetilde{\Lambda}_{m}$, coupled with a smooth flow on $M$. Since $M$ is compact, the behavior of the flows on the fibres is the only essential component in considering compactness properties of the total flow.

Claim 6.2. The vector field $\nabla F$ is smooth and has a globally defined flow on $\widetilde{\Lambda}$. 
The smoothness of $\nabla F$ follows from that of the functions $h_{m}$. Inequality (7) then implies that $\nabla F$ is sublinear in the fibre directions which yields the completeness of the gradient flow (see $[\mathbf{A M R}]$ ).

Claim 6.3. Critical points of $F_{m}$ on $\widetilde{\Lambda}_{m}$ are smooth.

This is a standard regularity result (see [HZ3, Lemma 5, p. 88]). It justifies the extension of domains since any critical point of $F$ must satisfy $\nabla F_{m}(z)=0$ for some $m \in M$ and so $\nabla F(z)=0$ implies that $z \in \Lambda$.

Claim 6.4. $F$ satisfies the Palais-Smale condition on $\widetilde{\Lambda}$, provided that $q$ is not an even integer.

For the sake of clarity we defer the proof of this claim to an appendix. We just mention here that the claim follows from the careful choice of the asymptotic quadratic behavior of the functions $h_{m}$.

6.2. Proof of Lemma 4.2. We use the Minimax Lemma to establish the existence of a critical point of $F$ with a positive critical value and so we recall the setting of this theory. Let $G$ be a $C^{1}$ function on a Hilbert manifold $L$ and let $\mathfrak{T}$ be a family of subsets $T \subset L$. Set

$$
c(G, \mathfrak{T})=\inf _{T \in \mathfrak{T}} \sup _{z \in T} G(z) .
$$

We then have the following (see, e.g., $[\mathbf{H Z 3}]$ ):

Lemma 6.5 (Minimax Lemma). Let the following properties hold for $G$ and $\mathfrak{T}$ :

1. G satisfies the Palais-Smale condition.

2. The gradient vector field of $G$ gives rise to a global flow $\psi^{t}$.

3. The family $\mathfrak{T}$ is positively invariant under the gradient flow, i.e., $\psi^{t}(T)$ $\in \mathfrak{T}$ for all $T \in \mathfrak{T}$ and $t \geq 0$.

4. $-\infty<c(G, \mathfrak{T})<\infty$.

Then there exists $z_{c} \in L$ such that

$$
\nabla G\left(z_{c}\right)=0 \text { and } G\left(z_{c}\right)=c(h, \mathfrak{T}) .
$$

Denoting the flow of the negative gradient field of $F$ by $\psi^{t}$ we must now define a $\psi^{t}$-invariant family of sets $\mathfrak{T}$ such that $0<c(F, \mathfrak{T})<\infty$. To achieve this, we extend the linking argument of [HZ1].

Proposition 6.6. Let $e_{N}^{+}$be a nonvanishing section of smooth loops in $E_{N}^{+}$ with $\left\|e_{N}^{+}(m)\right\|_{\frac{1}{2}, m}^{2}=2 \pi$ for all $m \in M$ and let $q$ be greater than a certain constant depending on $e_{N}^{+}$only. Then there exists a sufficiently large $\tau>0$ such that for all $m \in M$ the subsets

$$
\begin{aligned}
\Sigma_{m}=\left\{x^{-}+y^{-}+y^{0}+s e_{N}^{+}(m)\right. & \in \widetilde{\Lambda}_{m} \mid \\
& \left.\left\|x^{-}+y^{-}+y^{0}\right\|_{\frac{1}{2}, m} \leq \tau, \quad 0 \leq s \leq \tau\right\}
\end{aligned}
$$


satisfy $\left.F\right|_{\partial \Sigma_{m}} \leq 0$.

Proof. First we label the parts of $\partial \Sigma_{m}$ as follows

$$
\begin{aligned}
\sigma_{1} & =\{s=0\}, \\
\sigma_{2} & =\{s=\tau\}, \\
\sigma_{3} & =\left\{\left\|x^{-}+y^{-}+y^{0}\right\|_{\frac{1}{2}, m}=\tau\right\} .
\end{aligned}
$$

For $z=x^{-}+x^{+}+y^{-}+y^{0}+y^{+} \in \widetilde{\Lambda}_{m}$ recall that

$$
\begin{aligned}
F_{m}(z)= & \frac{1}{2}\left(\left\|x^{+}+y^{+}\right\|_{\frac{1}{2}, m}^{2}-\left\|x^{-}+y^{-}\right\|_{\frac{1}{2}, m}^{2}\right)-\int_{0}^{1} h_{m}(z(t)) d t \\
= & \frac{1}{2}\left(\left\|x^{+}\right\|_{\frac{1}{2}, m}^{2}+\left\|y^{+}\right\|_{\frac{1}{2}, m}^{2}\right) \\
& -\frac{1}{2}\left(\left\|x^{-}\right\|_{\frac{1}{2}, m}^{2}+\left\|y^{-}\right\|_{\frac{1}{2}, m}^{2}\right)-\int_{0}^{1} h_{m}(z(t)) d t .
\end{aligned}
$$

On $\sigma_{1}$ we have points of the form $z=x^{-}+y^{-}+y^{0}$ so that

$$
\left.F_{m}\right|_{\sigma_{1}}(z)=-\frac{1}{2}\left(\left\|x^{-}\right\|_{\frac{1}{2}, m}^{2}+\left\|y^{-}\right\|_{\frac{1}{2}, m}^{2}\right)-\int_{0}^{1} h_{m}\left(z_{1}(t)\right) d t \leq 0 .
$$

For the other parts of the boundary we need to employ the first half of inequality (6),

$$
h_{m}(z) \geq \frac{q}{2} \pi\|y\|_{m}^{2}-b .
$$

This yields

$$
\begin{aligned}
& \int_{0}^{1} h_{m}(z(t)) d t \\
& \geq \frac{q}{2} \pi\left(\int_{0}^{1}\left\|y^{-}(t)\right\|_{m}^{2} d t+\int_{0}^{1}\left\|y^{0}\right\|_{m}^{2} d t+\int_{0}^{1}\left\|y^{+}(t)\right\|_{m}^{2} d t\right)-b
\end{aligned}
$$

which when restricted to $\Sigma_{m}$ becomes

$$
\begin{aligned}
& \int_{0}^{1} h_{m}(z(t)) d t \\
& \geq \frac{q}{2} \pi\left(\int_{0}^{1}\left\|y^{-}(t)\right\|_{m}^{2} d t+\left\|y^{0}\right\|_{m}^{2}+s^{2} \int_{0}^{1}\left\|e_{N}^{+}(m)(t)\right\|_{m}^{2} d t\right)-b .
\end{aligned}
$$

Overall, on $\Sigma_{m}$ we now have

$$
F_{m}(z) \leq b-\frac{1}{2}\left\|x^{-}+y^{-}+y^{0}\right\|_{\frac{1}{2}, m}^{2}-s^{2}\left(\frac{q}{2} \pi \int_{0}^{1}\left\|e_{N}^{+}(m)(t)\right\|_{m}^{2} d t-\pi\right) .
$$

For our nonvanishing section $e_{N}^{+}$it is clear that there exists a real constant $c>0$ such that $\int_{0}^{1}\left\|e_{N}^{+}(m)(t)\right\|_{m}^{2} d t \geq c$ for all $m \in M$. We now choose $q$ to 
be greater than $\frac{2}{c}$. Then for $\tau$ large enough to satisfy both $\tau^{2}\left(\frac{q}{2} \pi c-\pi\right) \geq b$ and $\frac{1}{2} \tau^{2} \geq b$, we have $\left.F_{m}\right|_{\sigma_{2}, \sigma_{3}} \leq 0$.

Remark 6.7. One can show that $e_{N}^{+}$can be chosen in such a way that it suffices to take $q$ strictly greater than $2 l /(n-l)$.

Proposition 6.8. There exists a sufficiently small $\alpha>0$ such that for all $m \in M$ the subsets

$$
\Gamma_{m}=\left\{y^{+} \in \widetilde{\Lambda}_{m} \mid\left\|y^{+}\right\|_{\frac{1}{2}, m}^{2}=\alpha\right\}
$$

satisfy $\left.F\right|_{\Gamma_{m}} \geq \beta>0$ for some $\beta \in \mathbb{R}^{+}$.

Proof. Since the functions $h_{m}$ are equal to zero on a neighborhood of $T_{m} M \times$ $\{0\} \subset T_{m} W$, the functions

$$
\begin{aligned}
\beta_{m}: \widetilde{\Lambda}_{m} & \rightarrow \mathbb{R} \\
z & \mapsto \int_{0}^{1} h_{m}(z(t)) d t
\end{aligned}
$$

satisfy $\beta_{m}(0)=0, \beta_{m}^{\prime}(0)=0$, and $\beta_{m}^{\prime \prime}(0)=0$ for all $m$ in $M$. (Here "'”" denotes a fibrewise derivative.) Restricting to the spaces $E_{N}^{+}(m)$ we see then that

$$
\left.F_{m}\right|_{E_{N}^{+}(m)}(z)=\frac{1}{2}\left(\left\|y^{+}\right\|_{\frac{1}{2}, m}^{2}\right)+O\left(\left\|y^{+}\right\|_{\frac{1}{2}, m}^{3}\right) .
$$

For $\tau>\alpha$, each $\Sigma_{m}$ and $\Gamma_{m}$ intersect at $\sqrt{\frac{\alpha}{2 \pi}} e_{N}^{+}(m)$. Since $\Sigma_{m}$ and $\Gamma_{m}$ depend smoothly on $m$ we may extend them to form global subsets of the bundle $\widetilde{\Lambda}$. We denote these subsets as $\Sigma$ and $\Gamma$, and note that they also intersect (in each fibre). Since $\left.F\right|_{\partial \Sigma} \leq 0$ and $\left.F\right|_{\Gamma}>0$, we expect the image of $\Sigma$ under the negative gradient flow of $F$ to still intersect $\Gamma$.

Proposition 6.9. $\psi^{t}(\Sigma) \cap \Gamma \neq \emptyset$, for all $t \geq 0$.

Proof. Let $P^{-}, P^{0}$ and $P^{+}$be the projection maps corresponding to the splitting $\widetilde{\Lambda}=E^{-} \oplus E^{0} \oplus E^{+}$. Consider the maps

$$
\begin{aligned}
\varphi^{t}: \Sigma \rightarrow & E^{-} \oplus E^{0} \oplus \mathbb{R} e_{N}^{+} \\
(m, z) \mapsto & \left(\psi_{m}^{t}(m, z),\left(P^{-}+P^{0}\right) \psi_{z}^{t}(m, z)\right. \\
& \left.\quad+\left(\left\|\psi_{z}^{t}(m, z)\right\|_{\frac{1}{2}, m}^{2}-\alpha\right) e_{N}^{+}\left(\psi_{m}^{t}(m, z)\right)\right)
\end{aligned}
$$

where $z=z^{-}+z^{0}+s e_{N}^{+}(m) \in \Sigma_{m}$. Letting $Z$ be the zero section in $E^{-} \oplus E^{0} \oplus \mathbb{R} e_{N}^{+}$it follows easily that

$$
\psi^{t}(\Sigma) \cap \Gamma \neq \emptyset \Longleftrightarrow\left(\varphi^{t}\right)^{-1}(Z) \neq \emptyset .
$$


The maps $\varphi^{t}$ are smooth and we claim that they are also nonlinear Fredholm maps with index equal to zero. This is easily confirmed for $\varphi^{0}$ and so must also hold for $\varphi^{t}$ with $t>0$ sufficiently small. Then, since $\varphi^{t}=\left(\varphi^{\frac{t}{k}}\right)^{k}$ for any $k \in \mathbb{N}$, it also holds for arbitrary $t$.

One may also easily verify that $\varphi^{0}$ is transversal to $Z$ and that $\left(\varphi^{0}\right)^{-1}(Z)$ $=e_{N}^{+} \subset \Sigma \cap \Gamma$.

We now consider a fixed $t>0$. Note that if $\varphi^{t}$ is not transversal to $Z$, then the proof is complete since transversality must fail at some $(m, 0) \in Z$ with $\left(\varphi^{t}\right)^{-1}((m, 0)) \in \Sigma$. Hence, we may assume that $\varphi^{t}$ is transversal to $Z$. By the extension theorem of Smale (see [Sm, Theorem 3.1]) we can then perturb the homotopy $\varphi^{r}, r \in[0, t]$, from $\varphi^{0}$ to $\varphi^{t}$ to a transversal Fredholm homotopy

$$
\widetilde{\varphi}: \Sigma \times[0, t] \rightarrow E^{-} \oplus E^{0} \oplus \mathbb{R} e_{N}^{+}
$$

such that $\widetilde{\varphi}(\cdot, 0)=\varphi^{0}(\cdot)$ and $\widetilde{\varphi}(\cdot, t)=\varphi^{t}(\cdot)$. The sign of $F$ on $\partial \Sigma$ and $\Gamma$ ensures that $\left(\varphi^{s}\right)^{-1}(Z) \cap \partial \Sigma=\emptyset$ for all $s \geq 0$. Consequently, $(\widetilde{\varphi})^{-1}(Z) \cap$ $\{\partial \Sigma \times[0, t]\}=\emptyset$. With this, Theorem 3.3 of $[\mathbf{S m}]$ implies that $(\widetilde{\varphi})^{-1}(Z)$ is a $2 l+1$ dimensional submanifold of $\Sigma \times[0, t]$ with boundary equal to

$$
\left\{\left(\varphi^{0}\right)^{-1}(Z) \times\{0\}\right\} \coprod\left\{\left(\varphi^{t}\right)^{-1}(Z) \times\{t\}\right\} .
$$

Upon projecting to $M$ we see that these boundary components must generate the same homology class in $H_{2 l}\left(M ; \mathbb{Z}_{2}\right)$. It is easy to see that the first component generates the fundamental class and hence the second component must be nonempty.

Remark 6.10. A similar argument shows that $\psi_{t}(\Sigma) \cap \Gamma_{m} \neq \emptyset$ for any $m \in M$.

We now complete the proof of Lemma 4.2 and hence Theorem 1.2 with a direct application of the Minimax Lemma. Consider the family of subsets $\mathfrak{T}=\left\{\psi^{t}(\Sigma) \mid t \geq 0\right\}$. It is clearly positively invariant under the flow. Let

$$
c(F, \mathfrak{T})=\inf _{t \geq 0} \sup _{z \in \psi^{t}(\Sigma)} F(z) .
$$

By the previous propositions and the fact that $F$ takes bounded sets to bounded sets we have

$$
\beta \leq \inf _{z \in \Gamma} F(z) \leq \sup _{z \in \psi^{t}(\Sigma)} F(z) \leq \infty
$$

This implies that

$$
0<\beta \leq c(F, \mathfrak{T})<\infty
$$

and by the Minimax Lemma we have proven the existence of the desired critical point. 


\section{Appendix: The Palais-Smale condition for F.}

Proof of Claim 6.4: F satisfies the Palais-Smale condition on $\widetilde{\Lambda}$. We will make use of two distance functions on the bundle $\widetilde{\Lambda}$, which we now define. Consider the two fibrewise norms, \|\|$_{0, m}$ and \|\|$_{\frac{1}{2}, m}$, which are given by

$$
\|z\|_{\frac{s}{2}, m}=\left\|z_{0}\right\|_{m}^{2}+2 \pi \sum_{k \in \mathbb{Z}}|k|^{s}\left\|z_{k}\right\|_{m}^{2}
$$

for $s=0$ and $s=1$, respectively, where $z(t)=\sum_{k \in \mathbb{Z}} e^{k 2 \pi J(m) t} z_{k}$. Each of these norms yields a fibrewise metric which, when coupled with the base metric $g_{J}$, define an $L^{2}$ and an $H^{\frac{1}{2}}$ metric on $\widetilde{\Lambda}$. We are interested in the distance functions corresponding to these metrics which we will denote by $d_{L^{2}}$ and $d_{H^{\frac{1}{2}}}$.

Given a sequence $\left\{\left(m_{i}, z_{i}\right)\right\} \subset \widetilde{\Lambda}$ such that for the $H^{\frac{1}{2}}$-gradient we have

$$
\nabla F\left(m_{i}, z_{i}\right) \rightarrow 0
$$

with respect to the $H^{\frac{1}{2}}$ metric, we need to show that there exists a convergent subsequence with respect to $d_{H^{\frac{1}{2}}}$. (Since $M$ is compact we already know that there is a convergent subsequence $m_{i} \rightarrow m_{0} \in M$.)

In what follows we focus entirely on the fibre component of the gradient (equal to the gradient of the restriction to a fibre), which we again denote by $\nabla$. The fibre component, $\nabla F$, is a function $\widetilde{\Lambda} \rightarrow \widetilde{\Lambda}$, where we identify a tangent space to the fiber $\widetilde{\Lambda}_{m}$ with the fibre itself. Clearly, the norm of the fibre component does not exceed the norm of the gradient and hence (8) still holds for the fibre components.

Consider the special form that Condition (8) takes on $\widetilde{\Lambda}$. Recall that the functional $F$ is given as $F(m, z)=F_{m}(z)=\alpha_{m}(z)-\beta_{m}(z)$, where

$$
\alpha_{m}(z)=\int_{0}^{1} \frac{1}{2} \widetilde{g}_{J}(m)\left(-J_{m} \dot{z}, z\right) d t \quad \text { and } \quad \beta_{m}(z)=\int_{0}^{1} h_{m}(z(t)) d t .
$$

With respect to the fibrewise orthogonal splitting $z=z^{-}+z^{0}+z^{+}$(see Section 6.1), it is straightforward to check that

$$
\alpha_{m}(z)=\frac{1}{2}\left(\left\|z^{+}\right\|_{\frac{1}{2}, m}^{2}-\left\|z^{-}\right\|_{\frac{1}{2}, m}^{2}\right)
$$

and, since $\nabla$ denotes the fibre component of the gradient,

$$
\nabla \alpha_{m}(z)=z^{+}-z^{-} \text {. }
$$

As in [HZ3, Prop. 5 p. 86-7], one can also show that

$$
\nabla \beta_{m}(z)=j_{m}^{*} \nabla h_{m}(z),
$$

where $j_{m}^{*}$ is the formal adjoint of the inclusion $j_{m}: H^{\frac{1}{2}}\left(S^{1}, T_{m} W\right) \rightarrow L^{2}\left(S^{1}\right.$, $\left.T_{m} W\right)$ and is a compact map. Indeed, our second inequality (7) implies that 
$\nabla h_{m}$ takes bounded sets in $L^{2}\left(S^{1}, T_{m} W\right)$ to bounded sets so that the map $\nabla \beta: \widetilde{\Lambda} \rightarrow \widetilde{\Lambda}$ defined as $\nabla \beta(m, z)=\nabla \beta_{m}(z)$, is also compact.

We may now rewrite (8), in a slightly weakened form, as

$$
\left\|\left(z_{i}^{+}-z_{i}^{-}\right)-\left(\nabla \beta\left(m_{i}, z_{i}\right)\right)\right\|_{\frac{1}{2}, m_{i}} \rightarrow 0 .
$$

Assume first that the $\left\|z_{i}\right\|_{\frac{1}{2}, m_{i}}$ are bounded for an infinite subsequence of points. Without loss of generality we may assume that $\left(m_{i}, z_{i}\right)$ is this subsequence. By the compactness of the map $\nabla \beta$ we know that $\left\{\left(m_{i}, \nabla \beta\left(m_{i}, z_{i}\right)\right)\right\}$ is relatively compact. Thus, the sequence $\left\{\left(m_{i}, z_{i}^{+}-z_{i}^{-}\right)\right\}$has a convergent subsequence. Note that $z_{i}^{+}$and $z_{i}^{-}$are orthogonal to each other in $\widetilde{\Lambda}_{m_{i}}$. After passing if necessary to subsequences, this implies that each of the sequences $\left\{\left(m_{i}, z_{i}^{+}\right)\right\}$and $\left\{\left(m_{i}, z_{i}^{-}\right)\right\}$converges for the same subsequence of points $\left(m_{i}, z_{i}\right)$. As before, we may restrict our attention to this subsequence. Finally, since $\left\{\left(m_{i}, z_{i}^{0}\right)\right\}$ is a bounded sequence in a finite dimensional space it too has a convergent subsequence and the proof in this case is finished.

Looking for a contradiction, we assume that $\left\|z_{i}\right\|_{\frac{1}{2}, m_{i}}$ are unbounded for some infinite sequence on which we now focus. Set

$$
u_{i}=\frac{z_{i}}{\left\|z_{i}\right\|_{\frac{1}{2}, m_{i}}} \quad \text { and } \quad w_{i}=\frac{\nabla h_{m_{i}}\left(z_{i}\right)}{\left\|z_{i}\right\|_{\frac{1}{2}, m_{i}}} .
$$

The assumption (8) now takes the form

$$
\left\|u_{i}^{+}-u_{i}^{-}-j_{m_{i}}^{*} w_{i}\right\|_{\frac{1}{2}, m_{i}} \rightarrow 0 .
$$

By inequality (7), the sequence $\left\|w_{i}\right\|_{L^{2}, m_{i}}$ is bounded. Indeed,

$$
\left\|w_{i}\right\|_{L^{2}, m_{i}} \leq \frac{\left\|\nabla h_{m_{i}}\left(z_{i}\right)\right\|_{L^{2}, m_{i}}}{\left\|z_{i}\right\|_{\frac{1}{2}, m_{i}}} \leq \frac{\left\|\nabla h_{m_{i}}\left(z_{i}\right)_{i}\right\|_{L^{2}, m_{i}}}{\left\|z_{i}\right\|_{L^{2}, m_{i}}} \leq c_{1} .
$$

The compactness of the operators $j_{m_{i}}^{*}$ then implies that $\left\{\left(m_{i}, j_{m_{i}}^{*} w_{i}\right)\right\}$ is relatively compact in $\widetilde{\Lambda}$ with respect to $d_{H^{\frac{1}{2}}}$. Hence, the sequence $\left\{\left(m_{i}, u_{i}^{+}-\right.\right.$ $\left.\left.u_{i}^{-}\right)\right\}$is also relatively compact in $\widetilde{\Lambda}$ with respect to $d_{H^{\frac{1}{2}}}$. Just as above we then get a convergent subsequence

$$
\left(m_{i}, u_{i}\right) \rightarrow\left(m_{0}, u\right) .
$$

Note also that

$$
\|u\|_{\frac{1}{2}, m}=\lim \left\|u_{i}\right\|_{\frac{1}{2}, m_{i}}=1 .
$$

Now

$$
\begin{aligned}
d_{L^{2}}\left(\left(m_{i}, w_{i}\right),\left(m_{0}, \nabla Q_{m_{0}}(u)\right)\right) \leq & d_{L^{2}}\left(\left(m_{i}, w_{i}\right),\left(m_{i}, \nabla Q_{m_{i}}\left(u_{i}\right)\right)\right) \\
& +d_{L^{2}}\left(\left(m_{0}, \nabla Q_{m_{0}}(u)\right),\left(m_{i}, \nabla Q_{m_{i}}\left(u_{i}\right)\right)\right),
\end{aligned}
$$


where $Q_{m}(z)$ is the quadratic term in the definition of $h_{m}$. The second term on the right hand side goes to zero as $i \rightarrow \infty$ because $\nabla Q$ is continuous. As for the first term, we have

$$
d_{L^{2}}\left(\left(m_{i}, w_{i}\right),\left(m_{i}, \nabla Q_{m_{i}}\left(u_{i}\right)\right)\right)=\frac{1}{\left\|z_{i}\right\|_{\frac{1}{2}, m_{i}}}\left\|\nabla h_{m_{i}}\left(z_{i}\right)-\nabla Q_{m_{i}}\left(z_{i}\right)\right\|_{L^{2}, m_{i}} .
$$

However, by the construction of $h_{m}$, the difference $h_{m}-Q_{m}$ has compact support, and hence, as is easy to see, $\left\|\nabla h_{m}-\nabla Q_{m}\right\|_{L^{2}}$ is bounded on $\widetilde{\Lambda}$. Thus the first term also goes to zero and

$$
\left(m_{i}, w_{i}\right) \rightarrow\left(m_{0}, \nabla Q_{m_{0}}(u)\right)
$$

with respect to $d_{L^{2}}$. This means that

$$
\left(m_{i}, j_{m_{i}}^{*} w_{i}\right) \rightarrow\left(m_{0}, j_{m_{0}}^{*} \nabla Q_{m_{0}}(u)\right)
$$

with respect to $d_{H^{\frac{1}{2}}}$. Accordingly, $u$ satisfies

$$
u^{+}-u^{-}-j_{m_{0}}^{*}\left(\nabla Q\left(m_{0}, u\right)\right)=0 .
$$

This is equivalent to

$$
J_{N}\left(m_{0}\right) \dot{u}_{N}=q \pi u_{N} \quad \text { and } \quad u_{T}=0 .
$$

However, $u_{N} \rightarrow J_{N}\left(m_{0}\right) \dot{u}_{N}$ is a self adjoint map on $E_{N}\left(m_{0}\right)$ with spectrum $\{2 \pi \mathbb{Z}\}$. Since $q$ is not a positive even integer, this forces $u_{N}=0$ and we get a contradiction to $\|u\|_{\frac{1}{2}, m_{0}}=1$.

\section{References}

[AMR] R. Abraham, J. Marsden and T. Ratiu, Manifolds, Tensor Analysis, and Applications, Second edition, Applied Mathematical Sciences, 75, Springer-Verlag, New York-Berlin, 1988, MR 89f:58001, Zbl 0875.58002.

[Ar] V.I. Arnold, On some problems in symplectic topology, in 'Topology and GeometryRochlin Seminar', O.Ya. Viro (Editor), Lect. Notes in Math., 1346, Springer, 1988, 1-5, MR 90d:58036, Zbl 0655.58002.

[Bo] M. Bottkol, Bifurcation of periodic orbits on manifolds and Hamiltonian systems, J. Diff. Eq., 37 (1980), 12-22, MR 82d:58061, Zbl 0476.58016.

[CZ] C.C. Conley and E. Zehnder, Birkhoff-Lewis fixed point theorem and a conjecture of V.I. Arnold, Inven. Math., 73 (1983), 33-49, MR 85e:58044, Zbl 0516.58017.

[FR] E.R. Fadell and P.H. Rabinowitz, Generalized cohomological index theories for Lie group actions and an application to bifurcation problems for Hamiltonian systems, Invent. Math., 45 (1978), 48-67, MR 57 \#17677, Zbl 0403.57001.

[FHV] A. Floer, H. Hofer and C. Viterbo, The Weinstein conjecture on $P \times \mathbb{C}^{l}$, Math. Z., 203 (1990), 469-482, MR 91b:58064, Zbl 0666.58019.

[Gi1] V.L. Ginzburg, New generalizations of Poincaré's geometric theorem, Funct. Anal. Appl., 21(2) (1987), 100-106, MR 89f:58057, Zbl 0656.58027. 
[Gi2] _ On closed trajectories of a charge in a magnetic field. An application of symplectic geometry, in 'Contact and Symplectic Geometry', C.B. Thomas (Editor), Publications of the Newton Institute, Cambridge University Press, Cambridge, (1996), 131-148, MR 97j:58128, Zbl 0873.58034.

[Gi3] On the existence and non-existence of closed trajectories for some Hamiltonian flows, Math. Z., 223 (1996), 397-409, MR 97i:58144, Zbl 0862.58020.

[GK] V.L. Ginzburg and E. Kerman, Periodic orbits in magnetic fields in dimensions greater than two, in 'Geometry and topology in dynamics' (Winston-Salem, NC, 1998/San Antonio, TX, 1999), 113-121, Contemp. Math., 246, AMS, Providence, RI, (1999), MR 2000k:37087, Zbl 0948.37045.

[HZ1] H. Hofer and E. Zehnder, Periodic solutions on hypersurfaces and a result by C.Viterbo, Inven. Math., 90 (1987), 1-9, MR 89a:58040, Zbl 0631.58022.

[HZ2] _ A new capacity for symplectic manifolds, Analysis et cetera, P. Rabinowitz and E. Zehnder (Editors), (1990), 405-428, MR 91b:58069, Zbl 0702.58021.

[HZ3] Symplectic Invariants and Hamiltonian Dynamics, Birkhäuser, Basel, (1994), MR 96g:58001, Zbl 0805.58003.

[Ji] M.-Y. Jiang, Periodic solutions of Hamiltonian systems on hypersurfaces in a torus, Manuscripta Math., 85 (1994), 307-321, MR 95j:58142, Zbl 0819.58031.

[Ke] E. Kerman, Periodic orbits of Hamiltonian flows near symplectic critical submanifolds, IMRN, 17 (1999), 953-969, MR 2001g:37111, Zbl 0958.37041.

[LM] F. Lalonde and D. McDuff, Hofer's $L^{\infty}$-geometry: Energy and stability of flows I, II, Invent. Math., 122 (1995), 1-69, MR 96h:58063, Zbl 0844.58020, Zbl 0844.58021.

[Ly] A. Lyapunov, Problème générale de la stabilite du mouvement, Ann. Fac. Sci Toulouse, 2 (1907), 203-474.

[Ma] L. Macarini, Magnetic flows, Hofer-Zehnder capacity and the Kaluza-Klein metric. Preprint 2000.

[Mo] J. Moser, Periodic orbits near an equilibrium and a Theorem by Alan Weinstein, Comm. Pure and Appl. Math., 29 (1976), 727-747, MR 57 \#7672, Zbl 0346.34024.

[Po] L. Polterovich, Geometry on the group of Hamiltonian diffeomorphisms, Proceedings of the I.C.M., II (Berlin 1998), 401-410, MR 2000c:37120, Zbl 0909.58004.

[Sm] S. Smale, An infinite dimensional version of Sard's theorem, Amer. J. Math., 4 (1965), 861-866, MR 32 \#3067, Zbl 0143.35301.

[Vi] C. Viterbo, A proof of Weinstein's conjecture in $\mathbb{R}^{2 n}$, Ann. Inst. H. Poincaré Anal. Non Linéaire, 4 (1987), 337-356, MR 89d:58048, Zbl 0631.58013.

[We1] A. Weinstein, Perturbation of periodic manifolds of Hamiltonian systems, Bulletin Amer. Math. Soc., 77 (1971), 814-818, MR 43 \#6949, Zbl 0218.58006.

[We2] _ Symplectomorphisms and their Lagrangian Submanifolds, Adv. Math., 6 (1971), 329-346, MR 44 \#335, Zbl 0213.48203.

[We3] , Normal modes for non-linear Hamiltonian systems, Inven. Math., 20 (1973), 377-410, MR 48 \#9761, Zbl 0264.70020. 
[We4] _ Critical point theory symplectic geometry and Hamiltonian systems, in 'Proceedings of the 1983 Beijing Symposium on Differential Geometry and Differential Equations', S.T. Liao and S.S. Chern (Editors), Science Press, Beijing, China, (1986), 261-289, MR 88g:58070.

Received December 17, 2000. The work is partially supported by the NSF and by the faculty research funds of the University of California, Santa Cruz.

Department of Mathematics

University of California, Santa Cruz

SANTA CRUZ, CA 95064

E-mail address: ginzburg@math.ucsc.edu

The Fields Institute

222 College Street

TORONTO, ONTARIO

CANADA M5T 3J1

E-mail address: ekerman@fields.utoronto.ca 